\title{
Boundary-element analysis of magnetic polarization tensor for metallic cylinder
}

\author{
Zhongwen Jin', Ganghua Qin', Haidong Fan', Ruochen Huang ${ }^{2, *}$, Ziqi Chen², Qian Zhao ${ }^{3}$, \\ Mingyang Lu², Zhijie Zhang ${ }^{4}$, Anthony Peyton ${ }^{2}$, Wuliang Yin ${ }^{2}$
}

\author{
${ }^{1}$ Zhejiang Energy R \& D Institute Co., Ltd, Hangzhou, Zhejiang, 310003 China \\ ${ }^{2}$ School of Electrical and Electronic Engineering, University of Manchester, Manchester, M13 9PL UK \\ ${ }^{3}$ College of Engineering, Qufu Normal University, Shandong, 273165 China \\ ${ }^{4}$ School of Instrument and Electronics, North University of China, Taiyuan, Shanxi, 030051 China \\ *Corresponding author: Ruochen Huang (ruochen.huang@manchester.ac.uk)
}

\begin{abstract}
The magnetic polarization tensor has a promising capability of determining the geometry and material properties of metallic samples. In this paper, a novel computation method is proposed to estimate the magnetic polarization tensors for the metallic samples using the boundary element method. In this method, the metallic sample is placed in a uniformly distributed magnetic field. Based on assumptions that the excitation frequency and/or the conductivity of the sample is very high, the metallic sample is regarded as a perfect electrical conductor (PEC). Therefore, the scattered field at a certain distance can be simulated. By utilising the boundary element method, the magnetic polarization tensor can be derived from the simulated scattered field. The theoretical calculation is presented and simulations and experiments have been carried out to validate the proposed method. The results from the simulation are matched with the analytical solution for the case of sphere samples. Moreover, there is a good agreement between the simulation results and the experimental results for the copper cylindrical samples.
\end{abstract}

INDEX TERMS Boundary element method (BEM), magnetic polarization tensor, magnetic induction, dipole approximation.

\section{INTRODUCTION}

Non-destructive testing (NDT) raises an exciting possibility and is widely applied in many industrial fields including aerospace, rail transit and pipeline testing [1-10]. In recent years, researches have been dedicated in utilising NDT method for the purpose of metal detection. It contains various category methods which are frequently used with many applications, i.e., acoustic emission (AE) [11-12], ultrasonic testing (UT) [13-14] and eddy current testing (ECT) [15-16]. As a non-contact method, the magnetic field would be disturbed by the presence of the metal sample. Taking the advantage of this principle, it can monitor the presence of metal for food quality assessment and landmine clearance in real time. Compared with the $\mathrm{x}$-ray inspection system using eddy current coil, Bick et.al proposed the metal detector of using the HTS SQUID magnetometer to detect the stainlesssteel particles with higher sensitivity [17]. It shows that the proposed system was highly sensitive compared with conventional coil system under the condition of premagnetization. The limit of the system is that it can detect the
SS316 hypodermic needle piece with the length of $0.5 \mathrm{~mm}$ and a diameter of $650 \mu \mathrm{m}$ and a weight of around $0.6 \mathrm{mg}$. A digital procession algorithm using dual channels was proposed by Bai et al. to distinguish the metal and the product [18]. The design of sensor detector to cancel the coupling between the coils and balance the background field gives good performance in differentiating the landmine in mineralized soil [19-20]. However, it is still a challenge for researchers to classify the harmful metal object from a range of metal objects. Therefore, the magnetic polarization tensor, as a material characteristic parameter, plays an essential role in determining the metal samples.

When the magnetic field is given, the magnetic polarization tensor describes the variation of the magnetic field due to the existence of the metal sample, which contains distinctive information related to the shape, size, material of the tested sample and orientation of the given field. With the support of this parameter, the tested sample can be accurately detected and evaluated from various metallic samples. A range of mathematical formulations have been proposed and used to 
calculate magnetic polarization tensor for the purpose of metal detection. Khairuddin et al. proved the first order polarization tensor of the spheroids and ellipsoids can be used to classify different conductivities of spheroid/ellipsoid samples [21-22]. Khairuddin et al. using the hp-FEM method to calculate the polarization tensor of the sample. It is found that, for the threat and non-threat objects, there is a potential to use the normalised eigenvalues for real and imaginary parts of the polarization tensor to classify the objects [23]. Dekdouk et al. proposed a linear relationship between the dipole moment and the magnetic polarization tensor, and then the tensor can be reconstructed. Based on this model, the error of the calculated tensor is within $12 \%$ compared with the correct tensor [24]. An in-line scanning method by using rotation measurements is proposed to calculate the tensor with singular value decomposition method and regularized Gauss Newton method, and for the overdetermined system, it faces less impact on the determination of the inverse method [25].

In order to reconstruct the magnetic polarization tensor from the unknown field, a novel inversion method to estimate the magnetic polarization tensor for the metal sample is proposed. In this paper, the boundary element method is used and the metallic sample is assumed to be a perfect electrical conductor (PEC) due to the high excitation frequency / high conductivity of the metallic sample. A uniform magnetic field is generated to the PEC. Based on these settings, it is able to obtain the scattered field at a certain distance from the simulations with the aid of the boundary element method. Then the magnetic polarization tensor can be derived from the simulation field. The mathematical calculation and simulations of the scattered field are presented. The simulation results are compared with the analytical solution for the sphere case and there is a good agreement between the simulation results and the experimental results for the cases of cylindrical samples.

\section{Mathematical calculation of the tensor based on boundary element analysis}

\section{A. The scattered field based on the PEC assumption}

Boundary element method is a universal analysis method to simulate the unknown electromagnetic field. It reduces the computation burden where a three-dimensional problem is turned to a two-dimensional problem to obtain the solution. Besides, the test sample is a metallic non-magnetic object with high conductive. According to the skin depth equation shown in equation (1), when the excitation frequency is very high, the eddy current is mainly concentrated in the surface of the conductor and hardly goes deeper in the conductor. Due to its characteristic, the conductor sample is regarded as a perfect electrical conductor with no resistance.

$\delta=\frac{1}{\sqrt{\pi f \mu_{0} \mu_{r} \sigma}}$

Where: $\mathrm{f}$ is the excitation frequency, $\mu_{0}$ is the permeability of the air, $\mu_{r}$ is the relative permeability of the test sample and $\sigma$ is the conductivity of the test sample.
The test sample is placed in the free space, it is assumed that the magnetic field in the free space confirms to the quasi-static field. In the free space, the magnetic field, $\boldsymbol{H}_{a}$, is accorded with Ampere's law and Gauss's magnetic divergence law, which gives

$$
\begin{aligned}
& \nabla \times \boldsymbol{H}_{\boldsymbol{a}}=0 \\
& \nabla \cdot \mu_{0} \boldsymbol{H}_{\boldsymbol{a}}=0
\end{aligned}
$$

It can be noted that the magnetic field is non-rotational in the free space. Because of this fact, the magnetic field in the free space can be described by the magnetic scalar potential $\lambda_{a}$, which satisfies the Laplace equation shown in equation (4) and it should meet the condition shown in equation (5).

$\nabla^{2} \lambda_{a}=0$

$\left(\nabla^{2}+n_{1}^{2}\right) p_{1}\left(\boldsymbol{r}, \boldsymbol{r}^{\prime}\right)=-\delta\left(\boldsymbol{r}-\boldsymbol{r}^{\prime}\right)$

Assume that the magnetic scalar potential in the region 1 and region 2 (metal conductor and the free space) is defined as $\left(\nabla^{2}+n_{1}^{2}\right) \lambda_{1}(\boldsymbol{r})=q(\boldsymbol{r})$

$\left(\nabla^{2}++n_{2}^{2}\right) \lambda_{2}(\mathrm{r})=0$

Where: $p_{1}$ is a predefined function where the magnetic scaler potential should be under this condition. $n_{1}$ and $n_{2}$ are the parameters to satisfy the green's equation presented in equation (5).

Combine equation (5) and equation (6) by multiplying $p_{1}\left(\boldsymbol{r}, \boldsymbol{r}^{\prime}\right)$ and $\lambda_{1}(\boldsymbol{r})$ respectively, the equation turns to $-\left(\nabla^{2}+n_{1}^{2}\right) p_{1}\left(\boldsymbol{r}, \boldsymbol{r}^{\prime}\right) \lambda_{1}(\boldsymbol{r})+\left(\nabla^{2}+n_{1}^{2}\right) \lambda_{1}(\boldsymbol{r}) p_{1}\left(\boldsymbol{r}, \boldsymbol{r}^{\prime}\right)=$ $\delta\left(\boldsymbol{r}-\boldsymbol{r}^{\prime}\right) \lambda_{1}(\boldsymbol{r})+q(\boldsymbol{r}) p_{1}\left(\boldsymbol{r}, \boldsymbol{r}^{\prime}\right)$

Integrate the equation over region $1\left(V_{1}\right)$, which gives

$\lambda_{0}\left(\boldsymbol{r}^{\prime}\right)=\int_{S+S_{i n f}} d S \hat{n} \cdot\left[p_{1}\left(\boldsymbol{r}, \boldsymbol{r}^{\prime}\right) \nabla \lambda_{1}(\boldsymbol{r})-\right.$

$\left.\lambda_{1}(\boldsymbol{r}) \nabla p_{1}\left(\boldsymbol{r}, \boldsymbol{r}^{\prime}\right)\right]+\lambda_{1}\left(\boldsymbol{r}^{\prime}\right), \boldsymbol{r}^{\prime} \in V_{1}$

Substitute $r$ and $r^{\prime}$ with $r^{\prime}$ and $r$ respectively, which equals

$\lambda_{1}(\boldsymbol{r})=\lambda_{0}(\boldsymbol{r})-\int_{S} d S n \cdot\left[p_{1}\left(\boldsymbol{r}, \boldsymbol{r}^{\prime}\right) \nabla \lambda_{1}\left(\boldsymbol{r}^{\prime}\right)-\right.$

$\left.\lambda_{1}\left(\boldsymbol{r}^{\prime}\right) \nabla p_{1}\left(\boldsymbol{r}, \boldsymbol{r}^{\prime}\right)\right], \boldsymbol{r} \in V_{1}, \boldsymbol{r}^{\prime} \in V_{2}$

Therefore, from the above deviations, the Laplace equation can be written as a boundary integral equation, express as

$c \lambda_{a}(r)+\int_{\Gamma}\left[\lambda_{a}\left(r^{\prime}\right) \frac{\partial p}{\partial n}+p \frac{\partial \lambda_{a}\left(r^{\prime}\right)}{\partial n}\right] d \Gamma=\lambda_{0}(r)$

Where: $\mathrm{c}$ is a parameter related to the measurement position and $\Gamma$ is the surface of the test sample with the unit normal vector $n$ positive inwards, $\lambda_{0}(r)$ is the magnetic scalar potential produced by the primary magnetic field, $r$ and $r^{\prime}$ are the measurement position and the source position of the magnetic field respectively.

Since the test sample is a PEC, the magnetic field in the sample can be ignored and the magnetic field should be continuous between the surface of the test sample and the air, therefore the boundary condition should be met with the following equations,

$\left.\boldsymbol{H}_{\boldsymbol{a}}\right|_{\text {normal }}=\left.\mu_{r} \boldsymbol{H}_{\boldsymbol{c}}\right|_{\text {normal }}=0$

$\frac{\partial \lambda_{a}}{\partial n}=0$

Combine equations (11) - (13), the magnetic scalar potential can be expressed as

$c \lambda_{a}(\boldsymbol{r})+\int_{\Gamma}\left[\lambda_{a}\left(\boldsymbol{r}^{\prime}\right) \frac{\partial p\left(\boldsymbol{r}, \boldsymbol{r}^{\prime}\right)}{\partial \boldsymbol{n}}\right] d \Gamma=\lambda_{0}(\boldsymbol{r})$

Where: $\mathrm{c}$ is defined as 0.5 since the measurement position is fixed in the paper. 
Then, to simplify the whole system, it can be approximated as a linear system

$\sum_{j=1}^{N} A_{i j} \lambda_{a}^{i}\left(r_{j}\right)=\lambda_{0}^{i}\left(r_{i}\right) \quad i, j=1,2, \ldots, N$

Where: $\mathrm{N}$ is the point number from the discretization of the surface of the test sample.

Consequently, the magnetic scalar potential on the surface of the test sample can be obtained by solving the solution from equation (15).

After obtaining the potential value of the sample surface, the magnetic field in the free space can also be calculated by utilising equation (14) and can be expressed as

$\boldsymbol{H}_{a}(r)=\boldsymbol{H}_{0}+\boldsymbol{H}_{s}=\boldsymbol{H}_{0}+\left.\sum_{i=1}^{N}\left(\int_{\Gamma^{\prime}} \frac{\partial\left(\nabla p\left(\boldsymbol{r}, \boldsymbol{r}^{\prime}\right)\right)}{\partial \boldsymbol{n}} d \Gamma^{\prime}\right) \lambda_{a}^{i}\right|_{c}$

Where: $\boldsymbol{H}_{0}$ is the primary magnetic field, $\boldsymbol{H}_{s}$ is the scattered magnetic field, and $\left.\lambda_{a}^{i}\right|_{c}$ is the magnetic scalar potential on the surface of the test sample.

\section{B. Relationship between the tensor and the scattered field}

Once the magnetic field at a certain distance is obtained, the tensor of the test sample can be deduced. The magnetic polarization tensor is a 3 by 3 matrix, denoted as $M$, and the magnetic dipole moment, denoted as $\boldsymbol{m}$, is determined by the tensor and the magnetic field produced by the excitation coil, shown in following equations.

$$
M=\left(\begin{array}{lll}
M_{x x} & M_{x y} & M_{x z} \\
M_{y x} & M_{y y} & M_{y z} \\
M_{z x} & M_{z y} & M_{z z}
\end{array}\right)
$$$$
\boldsymbol{m}=M \cdot \boldsymbol{H}_{\boldsymbol{e}}
$$

Here $\boldsymbol{H}_{\boldsymbol{e}}$ is the incident magnetic field produced by the excitation coil at the test position. When the current is injected into the excitation coil with the magnitude of $I_{e}$, the voltage detected by the receiving coil can be given as

$V_{r x}=\left(\frac{i 2 \pi f \mu_{0}}{I_{e}}\right) \boldsymbol{H}_{\boldsymbol{r}} \cdot \boldsymbol{m}=\left(\frac{i 2 \pi f \mu_{0}}{I_{e}}\right)\left(\boldsymbol{H}_{\boldsymbol{r}} \cdot M \cdot \boldsymbol{H}_{\boldsymbol{e}}\right)$

Where: $\boldsymbol{H}_{\boldsymbol{r}}$ is the incident magnetic field at the test position produced by the receiving coil with the injected current magnitude of $I_{e}$.

It can be found that the magnetic field at the test sample is $\pi R_{e}{ }^{2} I_{e} \vec{z}$ when the receiving coil satisfies the following conditions:

a) The coil should be small and its radius is $R_{e}$.

b) It should be located on the Z-axis and parallel to the Zaxis.

c) The distance between the coil and the centre of the test sample is $(\sqrt[3]{2 \pi})^{-1}$.

Hence, apply the uniform magnetic field on the Z-axis and substitute $\boldsymbol{H}_{\boldsymbol{r}}$ with $\pi R_{e}{ }^{2} I_{e} \vec{Z}$, the received voltage equals

$V_{r x}=\frac{i 2 \pi f \mu_{0}}{I_{e}} \pi R_{e}{ }^{2} I_{e} \mathrm{M} \boldsymbol{H}_{e}=i 2 \pi f \mu_{0} \pi R_{e}{ }^{2} M_{z z}$

Besides, from Faraday's Law, the received voltage can also be calculated as

$V_{r x}=i 2 \pi f \mu_{0} \pi R_{e}^{2} \boldsymbol{H}_{s \boldsymbol{Z}}$

Combining equation (20) and equation (21), it can be noticed that $M_{z z}$ of the tensor is equal to the $\mathrm{z}$-component of the scattered magnetic field. Besides, from the similar deviations, $M_{x x}$ and $M_{y y}$ also have this property, which can be obtained by the $\mathrm{x}$ and $\mathrm{y}$ components of the scatted magnetic field respectively.

\section{The scattered field based on the dipole approximation}

Based on the assumption that the conductor is regarded as a magnetic dipole, the tensor then can be derived from the calculated scattered magnetic field. Considering a dipole, the dipole moment is presented as

$\boldsymbol{m}=m \vec{z}$

Then for the test cylindrical sample is placed at a certain distance with the value of $r$, the magnetic potential of the dipole is given in spherical coordinates,

$\boldsymbol{A}_{\text {dipole }}=\frac{\boldsymbol{m} \times \vec{r}}{r^{2}}=\frac{m \sin \theta}{r^{2}} \vec{\phi}$

$\boldsymbol{B}_{\text {dipole }}=\nabla \times \boldsymbol{A}_{\text {dipole }}=\frac{\vec{r}}{r \sin \theta} \frac{\partial}{\partial \theta} \sin \theta A_{\varphi}-\frac{\vec{\theta}}{r} \frac{\partial}{\partial r} r A_{\varphi}=$ $\frac{m \vec{r}}{r^{3} \sin \theta} \frac{\partial}{\partial \theta} \sin ^{2} \theta-\frac{m \sin \theta \vec{\theta}}{r} \frac{\partial}{\partial r} \frac{1}{r}=\frac{m \vec{r}}{r^{3} \sin \theta} 2 \sin \theta \cos \theta+$

$\frac{m \sin \theta \vec{\theta}}{r^{3}}=\frac{2 m \cos \theta}{r^{3}} \vec{r}+\frac{m \sin \theta}{r^{3}} \vec{\theta}=\frac{1}{r^{3}}[(3 m \cdot \vec{r}) \vec{r}-m]$

It can be seen from equation (24) that there is a reversed relationship between the scattered magnetic field due to the magnetic dipole and the distance between them. The scattered magnetic field attenuates as the distance increase. From this relationship, it can be used to assess the effect due to the size of the sample.

\section{Tensor reconstruction for cylindrical metals}

\section{A. Models}

In the simulation, the sample setup is shown in Fig. 1(a). As listed in Table I, the centre of the cylinder is placed on the origin of the coordinate system with the diameter of $5 \mathrm{~mm}$ and the height of $10 \mathrm{~mm}, 15 \mathrm{~mm}$ and $20 \mathrm{~mm}$ respectively. The height of the cylinder is parallel to the $\mathrm{Z}$-axis. The cylinder is assumed to be a PEC and a uniform magnetic field with the magnitude of $\boldsymbol{H}_{\mathbf{0}}$ is generated to the cylinder along the Z-axis. Besides, Fig. 1(b) shows the rotation direction of the cylinder. It rotates clockwise along the $\mathrm{x}$-axis from 0 to $360^{\circ}$ with an increment of $5^{\circ}$ for each rotation. In practical measurement, the measurement setup is shown in Fig. 2. The material of the conductor is copper with a conductivity of $59 \mathrm{MS} / \mathrm{m}$. The receiving coil is placed at a distance of $(\sqrt[3]{2 \pi})^{-1}$. The primary magnetic field is generated by an excitation coil placed far away to the test sample. In this case, the magnetic field in the vicinity of the cylinder can be assumed to be uniformly distributed. 


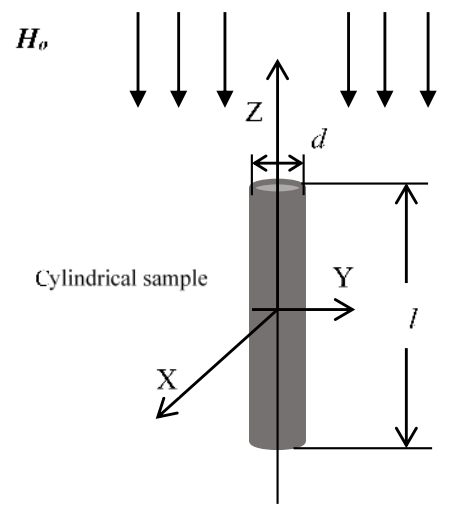

(a)

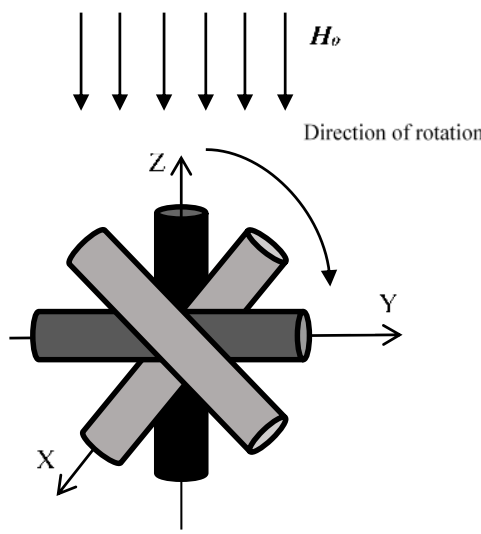

(b)

Figure 1. Numerical experimental setup (a) Cylinder dimension (b) Cylinder rotation

TABLE I

CYLINDRICAL SAMPLE PARAMETERS

\begin{tabular}{l|l}
\hline \hline Diameter of the cylinder $d$ & $5 \mathrm{~mm}$ \\
\hline \multirow{3}{*}{ Length of the cylinder $l$} & $10 \mathrm{~mm}$ \\
\cline { 2 - 2 } & $15 \mathrm{~mm}$ \\
\cline { 2 - 2 } & $20 \mathrm{~mm}$ \\
\hline Sample material & Copper \\
\hline
\end{tabular}

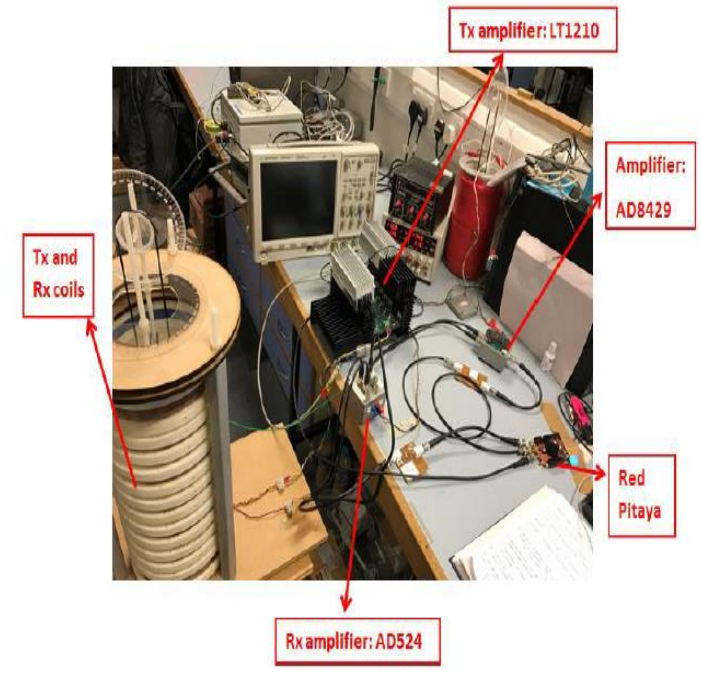

Figure 2. Measurement setup

\section{B. Test of the dipole approximation}

From the calculation, considering the dipole approximation, the strength of the scattered field attenuates with the increase of the distance between the test sample and the receiving coil and the product of them should be remain constant according to equation (24). When a small sample is placed $1 \mathrm{~m}$ away from the receiving coil shown in Fig. 3 (a), it can be seen that the product of the scattered field and the distance between the test sample and the receiving coil stays stable which equals $H_{\text {scattered }} \times r^{3}=$ constant

Consequently, for this case, the test sample can be assumed as a magnetic dipole. However, for the case that closely places the coil and the sample (i.e., the distance is $0.1 \mathrm{~m}$ ), as can be seen from Fig. 3(b), there is an error of $8 \%$ of the product. Moreover, a larger test sample is also used in the measurement. Fig. 3(c) shows the results of the product when a larger test sample is placed $1 \mathrm{~m}$ away from the receiving coil. Compared with Fig. 3(a), it can be concluded that the size of the test sample seldomly influence the scattered field. The scattered field is mainly influenced by the distance of the placement.

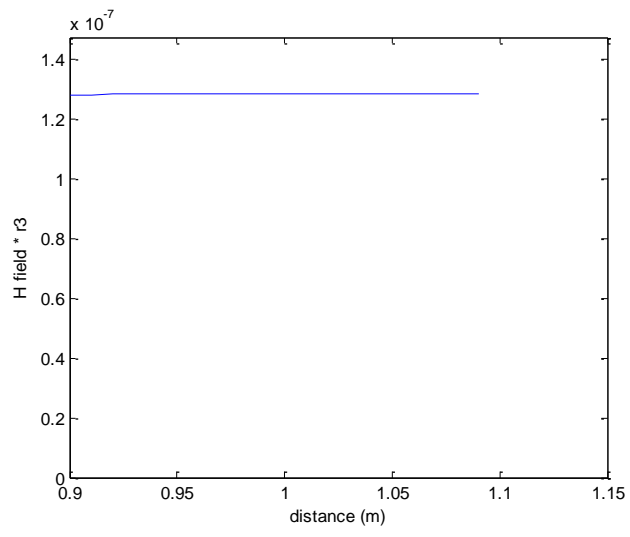

(a) 


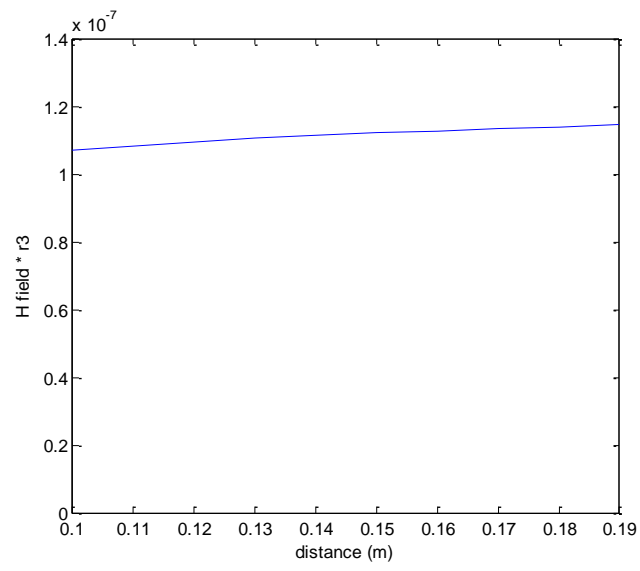

(b)

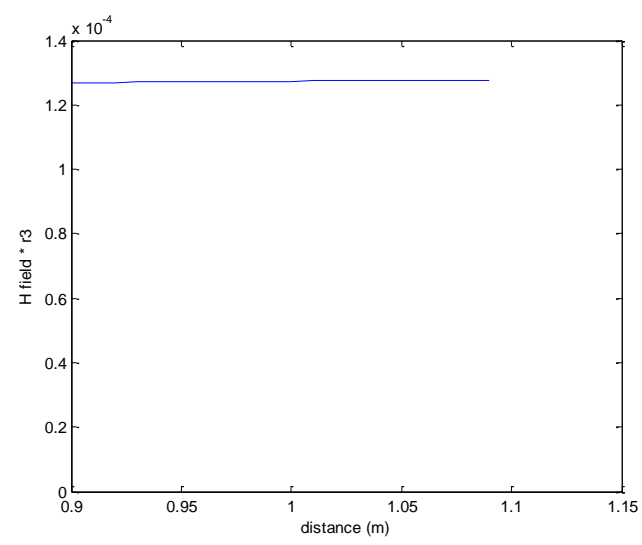

(c)

Figure 3. The product of the scattered field and the distance between the test sample and the receiving coil (a) a small test sample $1 \mathrm{~m}$ away from the receiving coil (b) a small test sample $0.1 \mathrm{~m}$ away from the receiving coil (c) a large test sample $1 \mathrm{~m}$ away from the receiving coil

\section{Tensor reconstruction}

As mentioned in mathematical calculation, the tensor can be obtained when the receiving coil is placed at the distance of $(\sqrt[3]{2 \pi})^{-1}$ with the radius of $R_{e}$. Referred to [26], when the sample is a sphere, the magnitude of the magnetic polarization tensor can be calculated by $2 \pi r^{3} I$. Compared with the analytical solution, there is only an error of $0.2 \%$ obtained from our BEM solver.

Figure 4 presents the magnetic field on the z-axis under various rotation angle from $0^{\circ}$ to $360^{\circ}$. It can be seen that, for different diameters and lengths of the cylindrical sample, the trend of the magnetic field is the same and it changes periodically with the rotation angle. The period of the change of the magnetic field is $180^{\circ}$. The magnetic field reaches maximum when the cylinder is vertical to the $\mathrm{Z}$-axis while it reaches minimum when the cylinder is parallel to the $\mathrm{Z}$-axis. As shown in Fig. 5, the magnetic polarization tensor of three different size of the copper cylinder can be measured under a range of the rotation angle at the frequency of $100 \mathrm{kHz}$. It can be seen that there is a good agreement for both simulations and measurements.

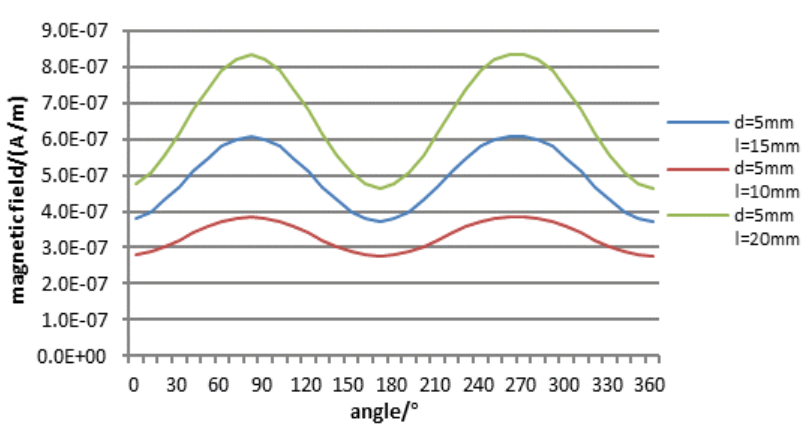

Figure 4. The magnetic field on Z-axis under different rotation angle

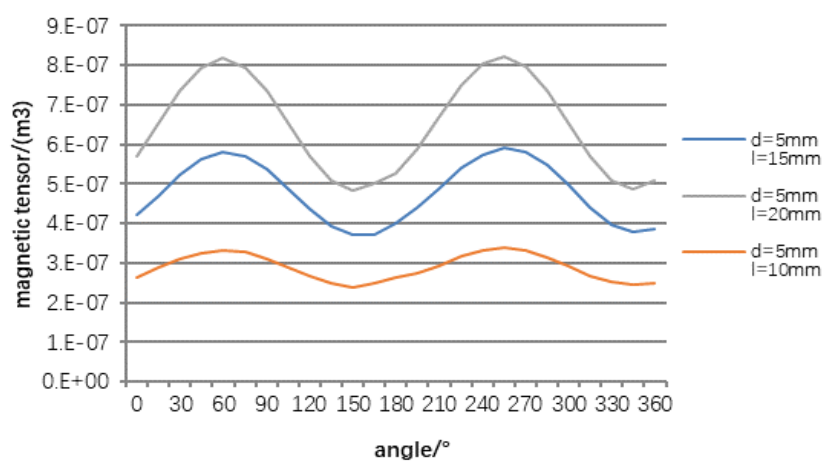

Figure 5. The magnetic polarization of the copper cylindrical sample under different rotation angle

\section{Conclusions}

In this paper, a reconstruction of the magnetic polarization tensor for cylindrical samples using the boundary element method is proposed. In this method, the excitation frequency is assumed to be high and the cylindrical metal is assumed as a PEC with high conductivity. Based on the dipole assumption, the magnetic tensor of the test sample can be estimated by using the relationship between the tensor and the scatted field. It is found that the scattered field is related to the distance of the receiving coil and the sample and, for the case that distance between them is large, the effect of the sample size is negligible. From the results from both the measurements, there is a good agreement between the simulation results of a PEC and the experimental results for the copper cylinders. From the results of the magnetic tensor, there is a potential to estimate the orientation of the cylinder by formulas.

\section{REFERENCES}

1. C. Garnier, M. L. Pastor, F. Eyma, and B. Lorrain, "The detection of aeronautical defects in situ on composite structures using Non Destructive Testing," Composite structures, vol. 93, no. 5, pp. 1328-1336, 2011.

2. F. Ciampa, P. Mahmoodi, F. Pinto, and M. Meo, "Recent Advances in Active Infrared Thermography for NonDestructive Testing of Aerospace Components", Sensors, vol. 18, no. 2, p. 609, Feb. 2018.

3. G. Yang, A. Tamburrino, L. Udpa, S. S. Udpa, Z. Zeng, Y. Deng, and P. Que, "Pulsed eddy-current based giant magnetoresistive system for the inspection of aircraft 
structures," IEEE transactions on magnetics, vol. 46, no. 3, pp. 910-917, 2009

4. W. Yin and K. Xu, "A Novel Triple-Coil Electromagnetic Sensor for Thickness Measurement Immune to Lift-Off Variations", IEEE Transactions on Instrumentation and Measurement, vol. 65, no. 1, pp. 164-169, Jan. 2016.

5. M. Lu, Y. Xie, W. Zhu, A.J. Peyton, and W. Yin, "Determination of the magnetic permeability, electrical conductivity, and thickness of ferrite metallic plates using a multi-frequency electromagnetic sensing system", IEEE Transactions on Industrial Informatics, vol. 15, no. 7, pp. 41114119, 2019

6. A. A. Carvalho, J. M. A. Rebello, M. P. V. Souza, L. V. S. Sagrilo, and S. D. Soares, "Reliability of non-destructive test techniques in the inspection of pipelines used in the oil industry," International journal of pressure vessels and piping, vol. 85, no. 11, pp. 745-751, 2008.

7. Y. M. Mao, and P. W. Que, "Application of Hilbert-Huang signal processing to ultrasonic non-destructive testing of oil pipelines," Journal of Zhejiang University-Science A, vol. 7, no. 2, pp. 130-134. 2006

8. C. Li, C. Chen, and K. Liao, "A quantitative study of signal characteristics of non-contact pipeline magnetic testing," Insight-Non-Destructive Testing and Condition Monitoring, vol. 57, no. 6, pp. 324-330, 2015.

9. Z. Orbán, and M. Gutermann, "Assessment of masonry arch railway bridges using non-destructive in-situ testing methods," Engineering Structures, vol. 31, no. 10, pp. 2287-2298, 2009.

10. S. S. Artagan, L. B. Ciampoli, F. D'Amico, A. Calvi, and F. Tosti, "Non-destructive assessment and health monitoring of railway infrastructures," Surveys in Geophysics, vol. 41, no. 3, pp. 447-483, 2020.

11. L. Yang, Y. C. Zhou, W. G. Mao, and C. Lu, "Real-time acoustic emission testing based on wavelet transform for the failure process of thermal barrier coatings," Applied physics letters, vol. 93, no. 23, 2008.

12. E. Siores, and A. A. Negro, "Condition monitoring of a gear box using acoustic emission testing," Materials evaluation, vol. 55 , no. 2, 1997.

13. A. M. T. Hassana and S. W. Jonesa, "Non-destructive testing of ultra high performance fibre reinforced concrete (UHPFRC): A feasibility study for using ultrasonic and resonant frequency testing techniques", Construction and Building Materials, vol. 35, pp. 361-367, October 2012

14. I. N. Prassianakis and N. I. Prassianakis, "Ultrasonic testing of non-metallic materials: concrete and marble", Theoretical and Applied Fracture Mechanics, vol. 42, no. 2, pp. 191-198, Nov. 2004.

15. W. Yin, and A. J. Peyton, "Thickness measurement of metallic plates with an electromagnetic sensor using phase signature analysis", IEEE Transactions on Instrumentation and Measurement, vol. 57, no. 8, pp. 1803-1807, 2008.

16. M. Lu, R. Huang, W. Yin, Q. Zhao, and A. Peyton, "Measurement of permeability for ferrous metallic plates using a novel lift-off compensation technique on phase signature," IEEE Sensors Journal, vol. 19, no. 17, pp. 7440-7446, 1 Sept.1, 2019.

17. M. Bick, P. Sullivan, D. L. Tilbrook, J. Du, S. Gnanarajan, K E. Leslie and C. P. Foley, "A SQUID-based metal detectorcomparison to coil and X-ray systems," Superconductor Science and Technology, vol. 18, no. 3, pp. 346, 2005

18. S. Bai and Y. Bai, "High Precision Algorithm of Metal Detector Based on Balance Coil", 2018 21st International Conference on Electrical Machines and Systems (ICEMS), Jeju, 2018, pp. 684-687, doi: 10.23919/ICEMS.2018.8549203.

19. W. R. Scott, "Broadband electromagnetic induction sensor for detecting buried landmines", 2007 IEEE International Geoscience and Remote Sensing Symposium, Barcelona, 2007, pp. 22-25, 2007.

20. J. Ge, W. Luo, H. Dong, X. Qiu, H. Liu, H. Wang, et al., "Characterization and reduction of background noise for the detection of plastic anti-personnel landmine in mineralized soil," Journal of Instrumentation, vol. 13, no. 09, p. 09023 2018.

21. T. A. Khairuddin, N. M. Yunos, Z. A. Aziz, T. Ahmad, and W. R. B. Lionheart, "Classification of materials for conducting spheroids based on the first order polarization tensor," Journal of Physics: Conference Series, vol. 890, no. 1, p. 012035, IOP Publishing, Sept. 2017.

22. T. A. Khairuddin, N. M. Yunos, and W. R. B. Lionheart, "Classification of Material and Type of Ellipsoid based on the First Order Polarization Tensor," Journal of Physics: Conference Series, vol. 1123, no. 1, p. 012035, IOP Publishing, Nov. 2018

23. T. K. Khairuddin, P. D. Ledger, and W. R. Lionheart, "Investigating the polarization tensor to describe and identify metallic objects," In Proc. of the World Congress on Engineering, vol. 1, pp. 122-127,2015.

24. B. Dekdouk, C. Ktistis, L. A. Marsh, D. W. Armitage, and A. J. Peyton, "Towards metal detection and identification for humanitarian demining using magnetic polarizability tensor spectroscopy," Measurement Science and Technology, vol. 26, no. 11,2015

25. Y. Zhao, W. Yin, C. Ktistis, D. Butterworth and A. J. Peyton, "Determining the Electromagnetic Polarizability Tensors of Metal Objects During In-Line Scanning," IEEE Transactions on Instrumentation and Measurement, vol. 65, no. 5, pp. 11721181, May 2016.

26. F. T. Ulaby, "Magnetostatics," Fundamentals of Applied Electromagnetics, 5th ed: Prentice Hall, 2006.

27. M. Lu, H. Xu, W. Zhu, L. Yin et al. "Conductivity Lift-off Invariance and measurement of permeability for ferrite metallic plates," NDT \& E International, vol. 95, pp. 36-44, Apr. 2018.

28. M. Lu, R. Huang, W. Yin, Q. Zhao, and A. Peyton, "Measurement of permeability for ferrous metallic plates using a novel lift-off compensation technique on phase signature," IEEE Sensors Journal, vol. 19, no. 17, pp. 7440-7446, 1 Sept.1, 2019.

29. M. Lu, et al. "Determination of the magnetic permeability, electrical conductivity, and thickness of ferrite metallic plates using a multi-frequency electromagnetic sensing system," IEEE Transactions on Industrial Informatics, vol. 15, pp. 4111-4119, 2019.

30. J.R.S. Avila, M. Lu et al. "Accurate measurements of plate thickness with variable lift-off using a combined inductive and capacitive sensor," NDT \& E International, vol. 110, pp. 102202, 2020

31. M. Lu, L. Yin, A. J. Peyton and W. Yin, "A novel compensation algorithm for thickness measurement immune to lift-off variations using eddy current method," IEEE

Transactions on Instrumentation and Measurement, vol. 65, no. 12, pp. 2773-2779, Dec. 2016.

32. M. Lu, X. Meng, W. Yin, Z. Qu, F. Wu, J. Tang, et al., "Thickness measurement of non-magnetic steel plates using a novel planar triple-coil sensor," NDT \& E International, vol. $107,2019$.

33. R. Huang, M. Lu, A. Peyton, and W. Yin, "Thickness measurement of metallic plates with finite planar dimension using eddy current method," IEEE Transactions on Instrumentation and Measurement, early access, 2020. Doi:10.1109/TIM.2020.2987413.

34. M. Lu et al. "Measurement of ferromagnetic slabs permeability based on a novel planar triple-coil sensor," IEEE Sensors J., vol. 20, no. 6, pp. 2904-2910, 2020.

35. M. Lu, W. Zhu, L. Yin, A. J. Peyton, W. Yin, and Z. Qu, "Reducing the lift-off effect on permeability measurement for magnetic plates from multifrequency induction data," IEEE Transactions on Instrumentation and Measurement, vol. 67, no. 1, pp. 167-174, Jan. 2018.

36. R. Huang, M. Lu et al, "Measurement of the radius of metallic plates based on a novel finite region eigenfunction expansion (FREE) method," IEEE Sensors Journal, vol. 20, pp. 15099 15106, 2020. Doi: 10.1109/JSEN.2020.3009443. 
37. R. Huang, M. Lu et al, "Measuring co-axial hole size of finitesize metallic disk based on a dual-constraint integration feature using multi-frequency eddy current testing," IEEE Transactions on Instrumentation and Measurement, vol. 70, pp. 1-7, 2020. Doi: 10.1109/TIM.2020.3026762.

38. M. Lu et al, "Thickness Measurement of Metallic Film Based on a High-Frequency Feature of Triple-Coil Electromagnetic Eddy Current Sensor," IEEE Transactions on Instrumentation and Measurement, vol. 70, pp. 1-8, 2020. Doi: 10.1109/TIM.2020.3027929.

39. M. Lu et al, "Measuring Lift-Off Distance and Electromagnetic Property of Metal Using Dual-Frequency Linearity Feature," IEEE Transactions on Instrumentation and Measurement, vol. 70, pp. 1-9, 2020. Doi: 10.1109/TIM.2020.3029348.

40. M. Lu et al, "Lift-off tolerant pancake eddy-current sensor for the thickness and spacing measurement of non-magnetic plates," IEEE Transactions on Instrumentation and Measurement, early access, 2020. Doi: 10.1109/TIM.2020.3033377.

41. M. Lu et al, "Inversion of distance and magnetic permeability based on material-independent and lift-off insensitive algorithms using eddy current sensor," IEEE Transactions on Instrumentation and Measurement, early access, 2020. Doi: 10.1109/TIM.2020.3036099.

42. W. Yin et al, "Measurements of Thickness for Metallic Plates With Co-Axial Holes Using a Novel Analytical Method With the Modified Integration Range," IEEE Access, vol. 8, pp. 198301 - 198306, 2020.

43. X. Meng, M. Lu et al, "Inversion of lift-off distance and thickness for non-magnetic metal using eddy current testing," IEEE Transactions on Instrumentation and Measurement, vol 70, 2020. Doi: 10.1109/TIM.2020.3038289.

44. M. Lu, X. Meng, et al, "Determination of surface crack orientation based on thin-skin regime using triple-coil drivepickup eddy-current sensor," IEEE Transactions on Instrumentation and Measurement, early access, 2020. Doi: 10.1109/TIM.2020.3044729.

45. Yin, W.; Tang, J.; Lu, M.; et al. An equivalent-effect phenomenon in eddy current non-destructive testing of thin structures. IEEE Access, 2019, 7, pp. 70296-70307.

46. Lu, M.; et al. Determination of Surface Crack Orientation Based on Thin-Skin Regime Using Triple-Coil Drive-Pickup Eddy-Current Sensor. IEEE Transactions on Instrumentation and Measurement, 2020, 70, pp. 1-9. DOI: 10.1109/TIM.2020.3044729

47. M. Lu, et al., "Prediction of the asymptotical magnetic polarization tensors for cylindrical samples using the boundary element method," In 2015 IEEE Sensors Applications Symposium (SAS), pp. 1-4. IEEE, 2015.

48. R. Huang, M. Lu, A. Peyton, and W. Yin, "A novel perturbed matrix inversion based method for the acceleration of finite element analysis in crack-scanning eddy current NDT," IEEE Access, vol. 8, pp. 12438-12444, 2020.

49. J. Tang et al., "A Novel Efficient FEM Thin Shell Model for Bio-Impedance Analysis," Biosensors, vol. 10, no. 6, pp. 69, 2020.

50. L. Chen, et al., "Textile Based Capacitive Sensor for Physical Rehabilitation via Surface Topological Modification," ACS Nano, vol. 14, no. 7, pp. 8191-8201, 2020. DOI: 10.1021/acsnano.0c01643

51. Z. Jin, et al., "Methods of Controlling Lift-off in Conductivity Invariance Phenomenon for Eddy Current Testing," IEEE ACCESS, vol. 8, pp. 2169-3536, 2020. DOI: 10.1109/ACCESS.2020.3007216.

52. J. Tang, et al., "Effect of frozen-thaw injury on cell membrane and bio-impedance," In 2020 IEEE International Instrumentation and Measurement Technology Conference (I2MTC), pp. 1-6. IEEE, 2020.

53. J. Tang, et al., "Bio-impedance spectroscopy for frozen-thaw of bio-samples: Non-contact inductive measurement and finite element (FE) based cell modelling," Journal of Food Engineering, vol. 272, pp. 109784, 2020.
54. H. Xu et al., "Imaging a weld cross-section using a novel frequency feature in multi-frequency eddy current testing," Insight-Non-Destructive Testing and Condition Monitoring, vol. 61, no. 12, pp. 738 - 743, 2019.

55. Y. Xie et al., "Novel Wearable Sensors for Biomechanical Movement Monitoring Based on Electromagnetic Sensing Techniques," IEEE Sensors Journal, vol. 20, no. 2, 2020. DOI: 10.1109/JSEN.2019.2943487

56. W. Yin et al., "Permeability invariance phenomenon and measurement of electrical conductivity for ferrite metallic plates," Insight-Non-Destructive Testing and Condition Monitoring, vol. 61, no. 8, pp. 472 - 479, 2019.

57. M. Lu et al., "A model for the triboelectric nanogenerator with inductive load and its energy boost potential," Nano Energy, vol. 63, pp. 103883, 2019.

58. M. Lu et al., "Forward solver for deep earth exploration and induction logging using custom built Edge-Element FEM technique," Acta Geologica Sinica, vol. 93, pp. 302-304, 2019.

59. L. Chen et al., "Whole System Design of Wearable Magnetic Induction Sensor for Physical Rehabilitation," Advanced Intelligent Systems, vol. 1, no. 1, pp. 1900037, 2019.

60. Y. X et al., "A self-powered radio frequency (RF) transmission system based on the combination of triboelectric nanogenerator (TENG) and piezoelectric element for disaster rescue/relief," Nano Energy, vol. 54, pp. 331-340, 2018.

61. W. Yin et al., "Custom edge-element FEM solver and its application to eddy-current simulation of realistic $2 \mathrm{M}$-element human brain phantom," Bioelectromagnetics, vol. 39, no. 8, pp. 604-616, 2018.

62. L. Yin et al., "Detection of corrosion pits based on an analytically optimised eddy current sensor," Insight-NonDestructive Testing and Condition Monitoring, vol. 60, no. 10, pp. 561-567, 2018.

63. W. Yin et al., "Acceleration of eddy current computation for scanning probes," Insight-Non-Destructive Testing and Condition Monitoring, vol. 60, no. 10, pp. 547-555, 2018.

64. W. Zhou et al., "Three-dimensional electromagnetic mixing models for dual-phase steel microstructures," Applied Sciences, vol. 8, no. 4, pp. 547-555, 2018.

65. M. Lu, et al., "Determining the magnetic permeability of ferrite steel strip by a custom inversion method," In Proc. 12th ECNDT, pp. 1-8. 2018.

66. J. Tang, et al., "Cellular structure analysis based on magnetic induction finite element method simulations and measurements," bioRxiv, pp. 275271, 2018. DOI: $10.1101 / 275271$

67. J.R.S. Avila, et al., "A novel dual modality sensor with sensitivities to permittivity, conductivity, and permeability," IEEE Sensors Journal, vol. 18, no. 1, pp. 356-362, 2017.

68. T. Yang, et al., "Level measurement for saline with a small surface area using high frequency electromagnetic sensing technique," Measurement, vol. 101, pp. 118-125, 2017.

69. Meng, X.; Lu, M.; Yin, W.; Bennecer, A.; Kirk, K.J. Evaluation of Coating Thickness Using Lift-Off Insensitivity of Eddy Current Sensor. Sensors 2021, 21, 419. https://doi.org/10.3390/s21020419

70. Lu, M.; Meng, X.; Huang, R.; Chen, L.; Peyton, A.; Yin, W Lift-off Invariant Inductance of Steels in Multi-Frequency Eddy-Current Testing. Preprints 2021, 2021040387 (doi: 10.20944/preprints202104.0387.v1).

71. Lu, M.; Meng, X.; Huang, R.; Chen, L.; Peyton, A.; Yin, W Lift-off Invariant Inductance of Steels in Multi-Frequency Eddy-Current Testing. NDT\&E International 2021. 